\title{
Acoustic signatures of the phase transitions in the antiferromagnet $U_{2} R h_{2} S n$
}

\author{
D. I. Gorbunov, ${ }^{1}$ A. V. Andreev, ${ }^{2}$ I. Ishii, ${ }^{3}$ K. Prokeš, ${ }^{4}$ T. Suzuki, ${ }^{3}$ S. Zherlitsyn, ${ }^{1}$ and J. Wosnitza ${ }^{1,5}$ \\ ${ }^{1}$ Hochfeld-Magnetlabor Dresden (HLD-EMFL) and Würzburg-Dresden Cluster of Excellence ct.qmat, Helmholtz-Zentrum \\ Dresden-Rossendorf, 01328 Dresden, Germany \\ ${ }^{2}$ FZU Institute of Physics, Czech Academy of Sciences, Na Slovance 2, 18221 Prague, Czech Republic \\ ${ }^{3}$ Department of Quantum Matter, ADSM, Hiroshima University, Higashi-Hiroshima 739-8530, Japan \\ ${ }^{4}$ Helmholtz-Zentrum Berlin für Materialien und Energie, Hahn-Meitner Platz 1, 14109 Berlin, Germany \\ ${ }^{5}$ Institut für Festkörper- und Materialphysik, TU Dresden, 01062 Dresden, Germany
}

(Received 12 November 2019; published 8 January 2020)

\begin{abstract}
We report on ultrasound measurements in a single crystal of the antiferromagnet $\mathrm{U}_{2} \mathrm{Rh}_{2} \mathrm{Sn}$ as a function of temperature and magnetic field. We find pronounced anomalies in the sound velocity at the Néel temperature, $25 \mathrm{~K}$, and at the field-induced spin-flop-like transition at $22.5 \mathrm{~T}$, which points to a strong magnetoelastic coupling. Additionally, we find that in the paramagnetic regime the temperature dependence of the magnetic susceptibility and the field dependences of the magnetization and sound velocity of transverse acoustic waves can be well described assuming a localized character of the $5 f$ electrons. Using this premise, the crystal-electric-field scheme of $\mathrm{U}_{2} \mathrm{Rh}_{2} \mathrm{Sn}$ has been determined.
\end{abstract}

DOI: 10.1103/PhysRevB.101.014408

\section{INTRODUCTION}

The structural, electronic, and magnetic properties of intermetallic compounds based on uranium are widely recognized to depend on the degree of the $5 f$ electron localization. The $5 f$ wave functions are on the border between localized, as generally are the $4 f$ states in lanthanides, and itinerant, as are the $d$ states of transition metals, and interact strongly with their environment [1-3]. The extended character of the $5 f$ states has two significant consequences. First, the defining electronic-structure quantities-the $5 f$ bandwidth, the screened intraatomic $f-f$ Coulomb interaction, and the spin-orbit interaction-are brought to the same energy scale in the eV range (the exchange interaction is usually much smaller and comparable to the external magnetic-field energy). Second, the degree of the localization of the $5 f$ states is easily variable. Uranium-based compounds are strongly susceptible to variations in external parameters such as temperature, magnetic field, pressure, and elemental substitution, and can be conveniently tuned into unique electronic states.

The strong spin-orbit interaction of uranium provides significant orbital polarization of the $5 f$ states and strongly couples the direction of the uranium magnetic moments and both the crystal and electronic structures. Magnetic anisotropy occurs in conjunction with elastic anisotropy: the direction of the uranium magnetic moments is determined by the bonding symmetry through a two-ion $5 f-5 f$ interaction, which, in the majority of cases, leads to extremely large anisotropy and prevalence of collinear magnetic structures [1]. The uranium moments tend to align as far as possible away from the nearest-neighbor U-U links.

Compounds with the general formula $\mathrm{U}_{2} T_{2} X$ ( $T$ is a $d$ metal, $X$ is a $p$ metal) make it possible to test the validity of this general picture. Here, one may compare isostructural
$\mathrm{U}_{2} \mathrm{Ni}_{2} \mathrm{Sn}$ and $\mathrm{U}_{2} \mathrm{Rh}_{2} \mathrm{Sn}$. Both materials are antiferromagnets below the Néel temperature, $T_{\mathrm{N}}=25 \mathrm{~K}$ [4-16]. For $\mathrm{U}_{2} \mathrm{Ni}_{2} \mathrm{Sn}$, the U-U distances within the basal plane of a tetragonal crystal structure, $3.575 \AA$, are substantially shorter than those along the $c$ axis, $3.693 \AA[4,6,11]$. Consequently, the magnetic moments align along the $c$ axis [9]. For $\mathrm{U}_{2} \mathrm{Rh}_{2} \mathrm{Sn}$, however, the shortest $\mathrm{U}-\mathrm{U}$ distances, $3.586 \AA$, are along the $c$ axis, while those in the basal plane are $3.622 \AA$ [10-12]. Nevertheless, the uranium moments also align along the $c$ axis.

$\mathrm{U}_{2} \mathrm{Rh}_{2} \mathrm{Sn}$ displays a field-induced phase transition for field applied along the easy magnetization direction [17-19]. The transition is reminiscent of a spin flop, whereby the magnetization continues to grow above the transition, showing a tendency toward saturation.

Here, we report on a high-field magnetoacoustic study of $\mathrm{U}_{2} \mathrm{Rh}_{2} \mathrm{Sn}$. Ultrasound technique is an extremely sensitive tool for detecting magnetoelastic couplings and structural phase transitions $[20,21]$. Changes in the acoustic properties originate mainly from the exchange-striction mechanism as sound waves change the positions of magnetic and nonmagnetic ions and renormalize the magnetic exchange interactions. For $\mathrm{U}_{2} \mathrm{Rh}_{2} \mathrm{Sn}$, we find pronounced anomalies in the sound velocity at the spontaneous and field-induced phase transitions. We use our experimental data to determine the crystal-electricfield scheme of this material.

\section{EXPERIMENTAL DETAILS}

A $\mathrm{U}_{2} \mathrm{Rh}_{2} \mathrm{Sn}$ single crystal was prepared by a modified Czochralski method as described in Ref. [12]. The lattice parameters of the tetragonal crystal structure (space group $P 4 / \mathrm{mbm})$ are $a=7.449(1) \AA$ and $c=3.586(1) \AA$. Backscattered Laue diffraction was used to check the singlecrystalline state and to orient the crystal for magnetization, specific-heat, and ultrasound measurements. 
Magnetization in static magnetic fields up to $14 \mathrm{~T}$ was measured using a commercial physical property measurement system (PPMS). The PPMS was also used for specific-heat measurements utilizing the relaxation method.

High-field magnetization was measured in pulsed magnetic fields up to $58 \mathrm{~T}$ by the induction method using a coaxial pickup coil system (a detailed description of the magnetometer can be found in Ref. [22]). Absolute values of the magnetization were calibrated using data obtained in static fields.

The field and temperature dependences of relative soundvelocity changes, $\Delta v / v$, were measured using a phase sensitive pulse-echo ultrasound technique [20,21]. A pair of piezoelectric transducers were glued to opposite surfaces of the sample in order to excite and detect acoustic waves. $\Delta v / v$ is proportional to the frequency change, $\Delta \omega / \omega$, phase change, $\Delta \phi / \phi$, and sample length change, $\Delta L / L: \Delta v / v=$ $\Delta \omega / \omega-\Delta \phi / \phi+\Delta L / L$. The last contribution can usually by neglected [20]. In static fields, we measured $\Delta v / v$ by fixing the phase and determining the frequency change. In pulsed fields, we fixed the frequency and analyzed the phase change of the acoustic wave.

We used $Z$ cut and $X$ cut $\mathrm{LiNbO}_{3}$ resonant transducers (Boston Piezo-Optics Inc.) to generate and detect longitudinal and transverse acoustic waves, respectively. We measured the longitudinal, $C_{11}(\boldsymbol{k}\|\boldsymbol{u}\|[100]), C_{33}$ $\left(\boldsymbol{k}\|\boldsymbol{u}\|\right.$ [001]), and transverse, $C_{44}\left(\boldsymbol{k}\|[100], \boldsymbol{u}\|\right.$ [001]), $C_{66}$ ( $\boldsymbol{k} \|$ [100], $\boldsymbol{u} \|$ [010]) acoustic modes. Here, $\boldsymbol{k}$ and $\boldsymbol{u}$ are the wave vector and polarization of acoustic waves, respectively. The absolute values of sound velocity for these modes at $2 \mathrm{~K}$ are $v_{11}=[3689 \pm 50] \mathrm{m} / \mathrm{s}, v_{33}=[3347 \pm 50] \mathrm{m} / \mathrm{s}, v_{44}=$ $[1883 \pm 50] \mathrm{m} / \mathrm{s}$, and $v_{66}=[1950 \pm 50] \mathrm{m} / \mathrm{s}$.

\section{RESULTS AND DISCUSSION}

The magnetic susceptibility, $\chi=M / H$, as well as the specific heat, $C$, of $\mathrm{U}_{2} \mathrm{Rh}_{2} \mathrm{Sn}$ show an anomaly at $T_{\mathrm{N}}=25 \mathrm{~K}$ [Figs. 1(a) and 1(b)]. The phase transition at the Néel temperature is also seen in the relative sound-velocity changes, $\Delta v / v$, due to magnetoelastic coupling [Fig. 1(c)]. The most pronounced changes at $T_{\mathrm{N}}$ are observed for the longitudinal mode $C_{11}$ for which $\Delta v / v$ displays a step.

The magnetization, $M$, shows a field-induced phase transition at $22.5 \mathrm{~T}$ for field applied along the [001] axis at $2 \mathrm{~K}$ [Fig. 2(a)]. Above the transition, $M$ changes the slope around $30 \mathrm{~T}$ but still continues to grow. No anomalies are observed for field applied in the basal plane. Neutron scattering shows that the uranium moments align along the [001] axis, while the rhodium moments form a noncollinear arrangement in the basal plane [12]. For isostructural $\mathrm{U}_{2} \mathrm{Ni}_{2} \mathrm{Sn}$, the [001] axis is also the easy magnetization direction [9]. However, for field applied along this direction, $\mathrm{U}_{2} \mathrm{Ni}_{2} \mathrm{Sn}$ displays three field-induced transitions.

We measured relative sound-velocity changes for the longitudinal $C_{11}$ and transverse $C_{44}$ acoustic modes for $\mathrm{U}_{2} \mathrm{Rh}_{2} \mathrm{Sn}$ in pulsed fields [Fig. 2(b)]. At $2 \mathrm{~K}, \Delta v / v$ for $C_{11}$ shows a sharp maximum at the magnetization jump followed by a broad minimum in the field range where the magnetization changes the slope. $\Delta v / v$ for $C_{44}$ displays a stepwise softening at the transition and changes the slope near $30 \mathrm{~T}$. Pronounced

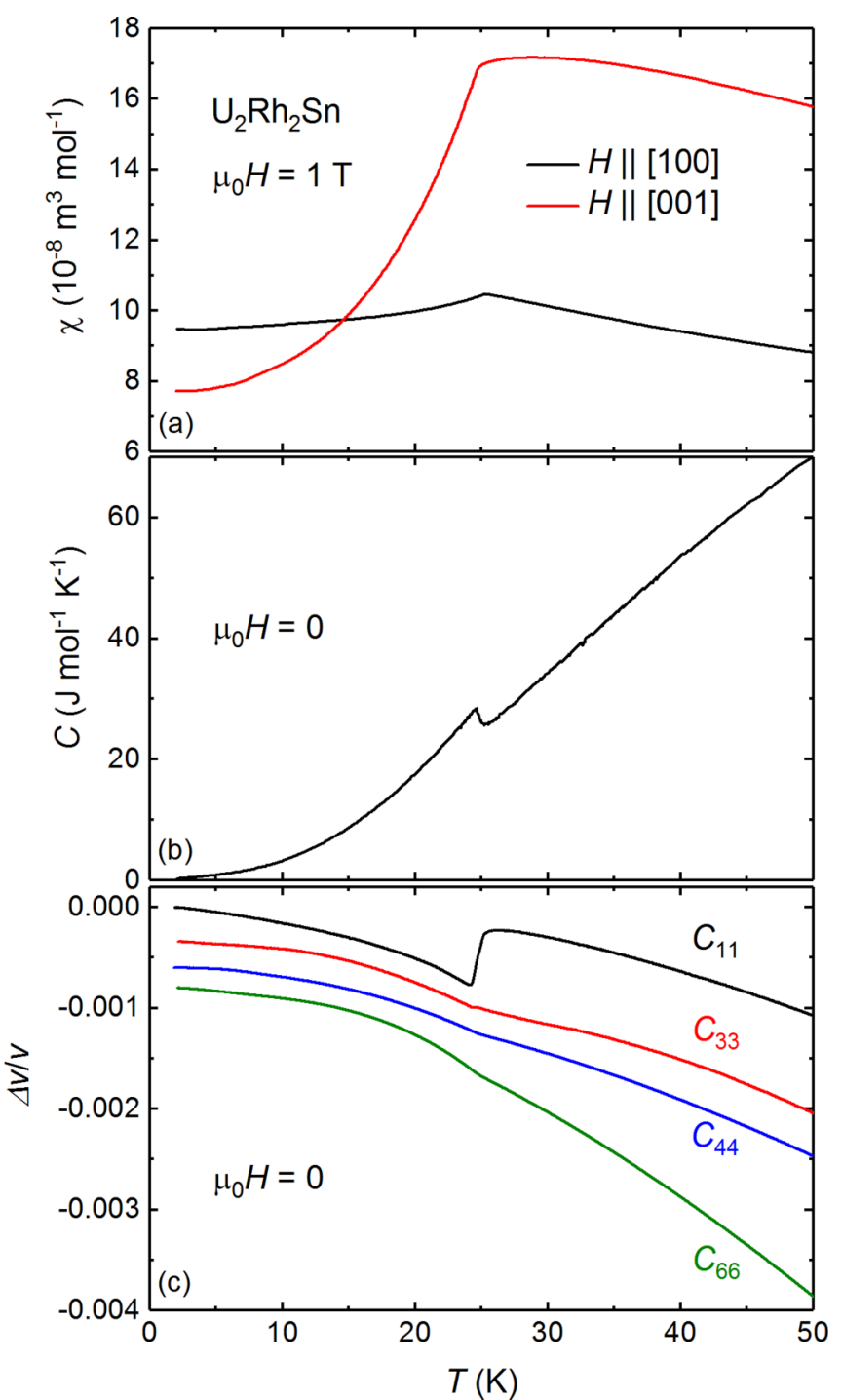

FIG. 1. Temperature dependences of (a) the magnetic susceptibility, $\chi=M / H$, in $1 \mathrm{~T}$; (b) the specific heat, $C$; and (c) the relative sound-velocity changes, $\Delta v / v$, for the acoustic modes $C_{11}, C_{33}, C_{44}$, and $C_{66}$ in zero field.

anomalies were also found in the acoustic properties at the field-induced transitions of $\mathrm{U}_{2} \mathrm{Ni}_{2} \mathrm{Sn}$ [9].

With increasing temperature, the anomaly in $\Delta v / v$ for $C_{11}$ at the spin-flop-like transition of $\mathrm{U}_{2} \mathrm{Rh}_{2} \mathrm{Sn}$ becomes more pronounced [Fig. 3(a)]. The broader character of the anomaly at higher temperatures corresponds to a broader jump in the magnetization [12]. Above $T_{\mathrm{N}}=25 \mathrm{~K}$, the phase transition is no longer observed in $\Delta v / v$. The stepwise anomaly in the relative sound-velocity changes for $C_{44}$ also gradually disappears with increasing temperature [Fig. 3(b)].

The magnetic phase diagram of $\mathrm{U}_{2} \mathrm{Rh}_{2} \mathrm{Sn}$ shows a good agreement between the critical fields of the spin-flop-like transition deduced from our magnetization and from the anomalies found in our sound-velocity measurements (Fig. 4).

For uranium-based intermetallic compounds, crystalelectric-field (CEF) effects are usually not so pronounced as for their lanthanide counterparts, which is due to the more extended character of the $5 f$ wave functions as compared 


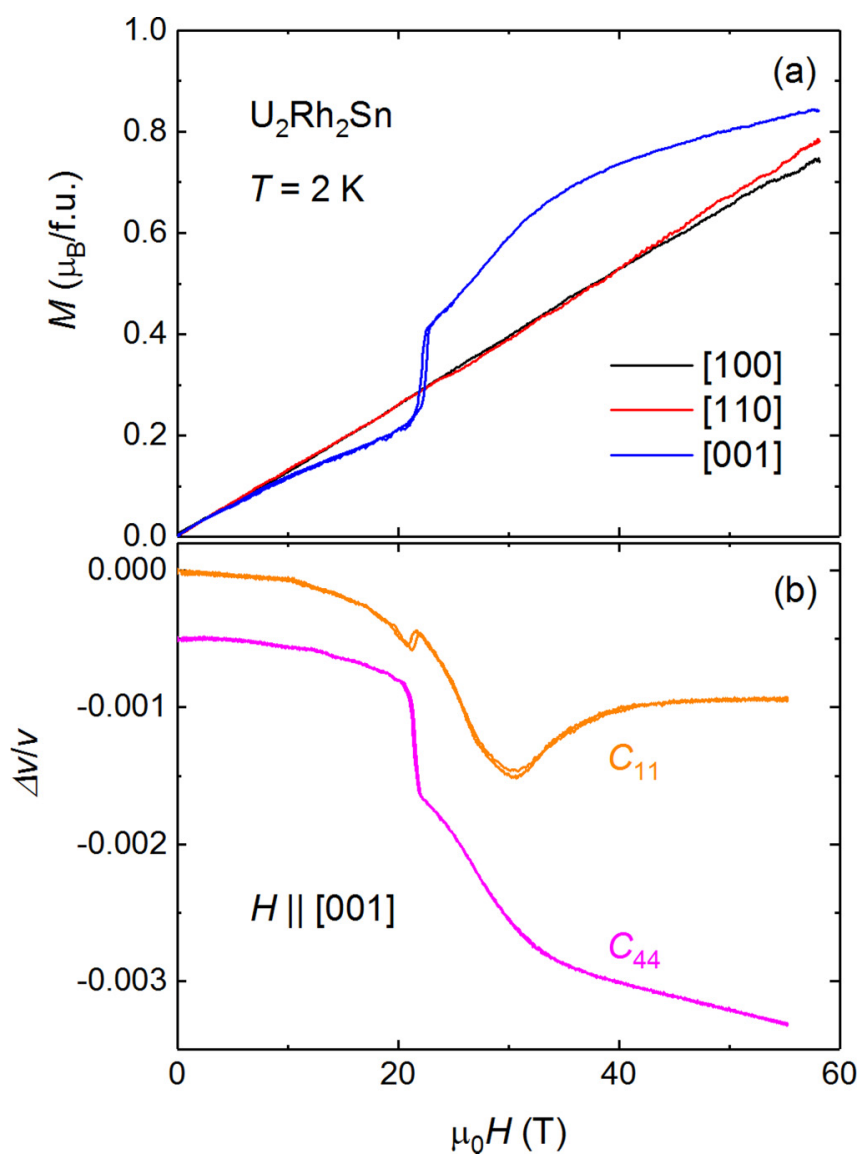

FIG. 2. (a) Magnetization, $M$, and (b) relative sound-velocity changes, $\Delta v / v$, for the acoustic modes $C_{11}$ and $C_{44}$ measured in pulsed magnetic fields up to $58 \mathrm{~T}$ at $2 \mathrm{~K}$.

to the $4 f$ wave functions. Nevertheless, some uranium-based materials show pronounced CEF effects, e.g., UNiSn and $\mathrm{UCu}_{2} \mathrm{Sn}[23,24]$. For a CEF analysis, we have to make an assumption about the state of the $5 f$ electrons in $\mathrm{U}_{2} \mathrm{Rh}_{2} \mathrm{Sn}$.

In uranium-based intermetallic compounds, the $5 f$ electrons frequently show both itinerant and localized behavior, depending on the temperature [2,25]. At high temperatures the magnetic properties can be described within a localized model, whereas at low temperatures they can be explained by itinerant $5 f$ electrons. $\mathrm{U}_{2} \mathrm{Rh}_{2} \mathrm{Sn}$ shows a relatively small ordered uranium magnetic moment and a small magnetic entropy [12]. This supports the picture of itinerant $5 f$ electrons in the low-temperature range, below $T_{\mathrm{N}}$. This situation likely changes at higher temperatures. One point in favor of this is that the effective magnetic moment deduced from a fit above $250 \mathrm{~K}$ approaches the value expected for a localized $\mathrm{U}^{3+}$ or $\mathrm{U}^{4+}$ moment [12]. We tested two $\mathrm{U}$ configurations for the analysis, $5 f^{2}\left(\mathrm{U}^{4+}\right)$ and $5 f^{3}\left(\mathrm{U}^{3+}\right)$, and found that the magnetic and elastic properties of $\mathrm{U}_{2} \mathrm{Rh}_{2} \mathrm{Sn}$ can be described much better by the model that assumes the $5 f^{2}$ configuration in the paramagnetic state.

We use the CEF model to reproduce our magneticsusceptibility, magnetization, and sound-velocity data for $C_{44}$ $[26,27]$. We consider the following effective Hamiltonian:

$$
H_{\text {eff }}=H_{\mathrm{CEF}}+H_{\mathrm{sQ}}+H_{\mathrm{QQ}}+H_{\mathrm{Zeeman}},
$$

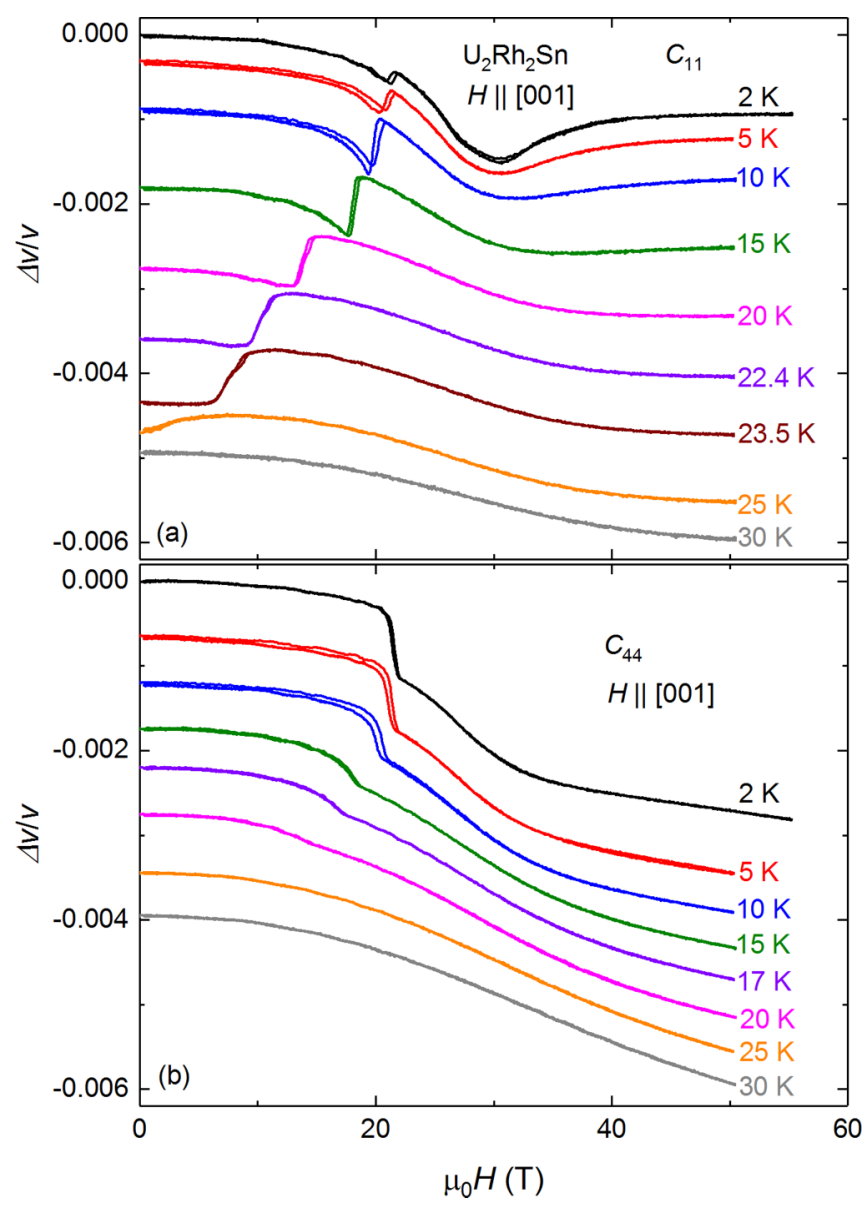

FIG. 3. Relative sound-velocity changes, $\Delta v / v$, for the acoustic modes (a) $C_{11}$ and (b) $C_{44}$ measured in pulsed magnetic fields up to $58 \mathrm{~T}$ between 2 and $30 \mathrm{~K}$.

where $H_{\mathrm{CEF}}, H_{\mathrm{sQ}}, H_{\mathrm{QQ}}$, and $H_{\mathrm{Zeeman}}$ are the $\mathrm{CEF}$, strainquadrupole, quadrupole-quadrupole, and Zeeman energy, respectively. In the tetragonal symmetry of $\mathrm{U}_{2} \mathrm{Rh}_{2} \mathrm{Sn}$, the CEF term is expressed as

$$
H_{\mathrm{CEF}}=B_{2}^{0} O_{2}^{0}+B_{4}^{0} O_{4}^{0}+B_{4}^{4} O_{4}^{4}+B_{6}^{0} O_{6}^{0}+B_{6}^{4} O_{6}^{4},
$$

where $B_{m}^{n}$ are crystal-field parameters and $O_{m}^{n}$ are Stevens' equivalent operators [28]. The strain-quadrupole interaction can be expressed as

$$
H_{\mathrm{sQ}}=-\sum_{i} g_{i} O_{i} \varepsilon_{i},
$$

where $g_{i}$ is the strain-quadrupole coupling constant, $O_{i}$ is the quadrupole operator, and $\varepsilon_{i}$ is the strain. Here, $i=y z, z x$ since the calculations of the elastic properties are performed for $C_{44}$. The quadrupole-quadrupole interaction is given by

$$
H_{\mathrm{QQ}}=-\sum_{i} g_{i}^{\prime}\left\langle O_{i}\right\rangle O_{i},
$$

where $g_{i}^{\prime}$ is the quadrupole-quadrupole coupling constant and $\left\langle O_{i}\right\rangle$ is a thermal average of the operator $O_{i}$. The Zeeman energy is

$$
H_{\text {Zeeman }}=-g_{j} \mu_{\mathrm{B}} J H,
$$




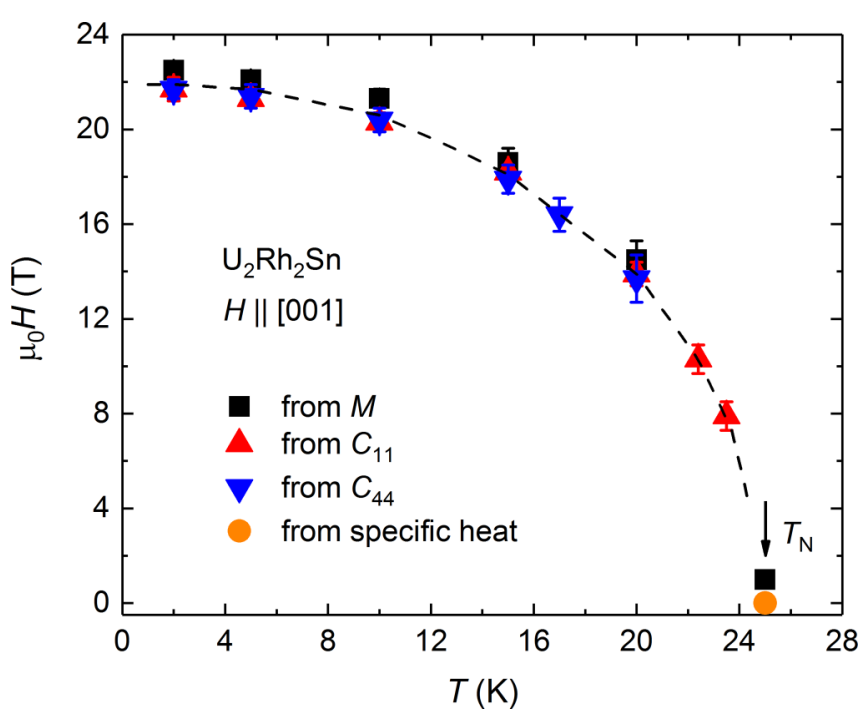

FIG. 4. Magnetic phase diagram of $\mathrm{U}_{2} \mathrm{Rh}_{2} \mathrm{Sn}$ for field applied along the [001] axis. The line is a guide to the eye.

where $g_{j}=0.8$ is the Lande factor and $J=4$ is the quantum number of the total angular momentum of a $\mathrm{U}^{4+}$ ion.

As a first step, we tested various combinations of the crystal-field parameters assuming singlet and doublet ground states to reproduce the $\chi$ vs $T$ and $M$ vs $H$ data. Then we calculated matrix elements of the nonperturbed Hamiltonian, $H_{\mathrm{CEF}}+H_{\text {Zeeman }}$, and included quadrupolar interactions as a perturbation. This allowed us to reproduce the field dependence of the elastic modulus $C_{44}$. Further details of such calculations can be found, e.g., in Refs. [29,30].

The best fit of the magnetic-susceptibility data [Fig. 5(a); the chi-squared value is 21.52 for the units $10^{-8} \mathrm{~m}^{3} \mathrm{~mol}^{-1}$ ] was obtained for the crystal-field parameters listed in
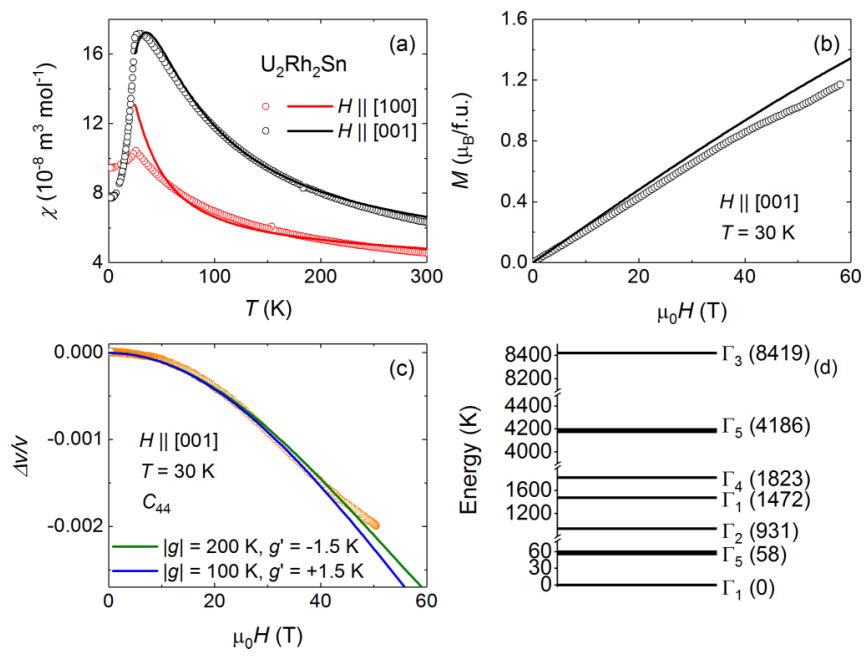

FIG. 5. (a) Temperature dependences of the magnetic susceptibility, $\chi$; (b) and (c) field dependences of the magnetization, $M$, and relative sound-velocity changes, $\Delta v / v$, for $C_{44}$; and (d) CEF level scheme of a $\mathrm{U}^{4+}$ ion in $\mathrm{U}_{2} \mathrm{Rh}_{2} \mathrm{Sn}$ obtained from the CEF parameters listed in Table I. In panels (a), (b), and (c), the symbols and solid lines represent the experimental and calculated data, respectively. In panel (d), the thin lines represent singlets and the thick lines represent doublets.
TABLE I. CEF parameters, $B_{m}^{n}(\mathrm{~K})$, for $\mathrm{U}_{2} \mathrm{Rh}_{2} \mathrm{Sn}$.

\begin{tabular}{lcccc}
\hline \hline$B_{2}^{0}$ & $B_{4}^{0}$ & $B_{4}^{4}$ & $B_{6}^{0}$ & $B_{6}^{4}$ \\
\hline$-39.9(5)$ & $-0.8(5)$ & $10.9(5)$ & $0.1(5)$ & $-0.5(5)$ \\
\hline \hline
\end{tabular}

Table I. We found a discrepancy between the experimental and calculated data for field applied along the [100] direction upon approaching the antiferromagnetic state for all tested combinations of the crystal-field parameters. This is likely related to changes in the localization of the $5 f$ electrons with decreasing temperature. We also obtained a reasonable agreement between experiment and theory for the field-dependent magnetization at $30 \mathrm{~K}$ [Fig. 5(b)].

Our analysis also reproduces the elastic softening observed for $C_{44}$ at $30 \mathrm{~K}$ [see Fig. 5(c) where two examples reproducing the data are shown]. Here, we varied the unknown parameters $g_{i}$ between 100 and $200 \mathrm{~K}$ and $g_{i}^{\prime}$ between -1.5 and $+1.5 \mathrm{~K}$ to obtain the best agreement with experiment. Remarkably, the use of both ferro- and antiferroquadrupolar interactions provides a satisfactory agreement with experiment. This likely indicates that quadrupole-quadrupole interactions are relatively weak but, nevertheless, have to be taken into account. Additional studies are needed to determine their role in the physics of $\mathrm{U}_{2} \mathrm{Rh}_{2} \mathrm{Sn}$.

In a tetragonal $\mathrm{CEF}$, the ninefold multiplet of a $\mathrm{U}^{4+}$ ion splits into five singlets and two doublets [Fig. 5(d)]. The ground state is a $\Gamma_{1}$ singlet and the first excited state is a $\Gamma_{5}$ doublet at $58 \mathrm{~K}$. The next excited levels have much higher energies.

The good agreement between experiment and theory in the paramagnetic state of $\mathrm{U}_{2} \mathrm{Rh}_{2} \mathrm{Sn}$ supports the general picture of $\mathrm{U}$ intermetallics; namely, that the $5 f$ electrons are more localized at high temperatures and more itinerant at low temperatures. In fact, this was proposed for $\mathrm{U}_{2} \mathrm{Rh}_{2} \mathrm{Sn}$ in Ref. [12] on the basis of magnetic-susceptibility data. The more localized nature of the $5 f$ electrons at high temperatures leads to well-defined CEF energy levels. As the $5 f$ electrons become more itinerant with decreasing temperature, a dispersion of the energy levels is likely to emerge affecting the separation between them. Here, the first excited doublet, $\Gamma_{5}$, at $58 \mathrm{~K}$ may shift to lower energies, close to $25 \mathrm{~K}$, causing the phase transition at $T_{\mathrm{N}}$.

\section{CONCLUSION}

Our study revealed pronounced anomalies in the elastic properties at the spontaneous and field-induced magnetic phase transitions of $\mathrm{U}_{2} \mathrm{Rh}_{2} \mathrm{Sn}$. We used our data to determine the crystal-electric-field scheme of this material. Our analysis shows that quadrupolar interactions have to be taken into account to reproduce the field-dependent sound-velocity data.

\section{ACKNOWLEDGMENTS}

The work was supported by Project No. 19-00925S of the Czech Science Foundation and by the Materials Growth and Measurement Laboratory [31] within the Program of Czech Research Infrastructures (Project No. LM2018096) and by JSPS KAKENHI Grants No. 17H06136, No. 18KK0078, 
and No. 19K03719. We acknowledge the support of HLD at HZDR, member of the European Magnetic Field Labora- tory (EMFL), and the excellence cluster ct.qmat (EXC 2147, Project ID 39085490).
[1] V. Sechovský and L. Havela, Magnetism of ternary intermetallic compounds of uranium, in Handbook of Magnetic Materials, edited by K. H. J. Buschow, Vol. 11 (Elsevier, Amsterdam, 1998).

[2] G. Zwicknagl and P. Fulde, The dual nature of $5 f$ electrons and the origin of heavy fermions in U compounds, J. Phys.: Condens. Matter 15, S1911 (2003).

[3] J. A. Mydosh and P. M. Oppeneer, Colloquium: Hidden order, superconductivity, and magnetism: The unsolved case of $\mathrm{URu}_{2} \mathrm{Si}_{2}$, Rev. Mod. Phys. 83, 1301 (2011).

[4] F. Bourée, B. Chevalier, L. Fournès, F. Mirambet, T. Roisnel, V. H. Tran, and Z. Zolnierek, Crystal and magnetic structures of $\mathrm{U}_{2} \mathrm{Ni}_{2} \mathrm{Sn}$ investigated by neutron diffraction and ${ }^{119} \mathrm{Sn}$ Mössbauer spectroscopy, J. Magn. Magn. Mater. 138, 307 (1994).

[5] L. Havela, V. Sechovský, P. Svoboda, H. Nakotte, K. Prokeš, F. R. de Boer, A. Seret, J. M. Winand, J. Rebizant, J. C. Spirlet, A. Purwanto, and R. A. Robinson, Magnetism in $\mathrm{U}_{2} \mathrm{~T}_{2} X$ compounds, J. Magn. Magn. Mater. 140-144, 1367 (1995).

[6] K. Kindo, T. Fukushima, T. Kumada, F. R. de Boer, H. Nakotte, K. Prokeš, L. Havela, V. Sechovský, A. Seret, J. M. Winand, J. C. Spirlet, and J. Rebizant, Electronic properties of $\mathrm{U}_{2} \mathrm{Ni}_{2} \mathrm{Sn}$, J. Magn. Magn. Mater. 140-144, 1369 (1995).

[7] R. P. Pinto, M. M. Amado, M. A. Salgueiro, M. E. Braga, J. B. Sousa, B. Chevalier, F. Mirambet, and J. Etourneau, Transport and magnetic properties of $\mathrm{U}_{2} \mathrm{Ni}_{2} \mathrm{Sn}$ and $\mathrm{U}_{2} \mathrm{Co}_{2} \mathrm{Sn}$, J. Magn. Magn. Mater. 140-144, 1371 (1995).

[8] K. Miliyanchuk, L. Havela, L. C. J. Pereira, A. P. Gonçalves, and K. Prokeš, Peculiarities of $\mathrm{U}_{2} \mathrm{~T}_{2} X$ hydrides, J. Magn. Magn. Mater. 310, 945 (2007).

[9] S. Mašková, A. V. Andreev, Y. Skourski, S. Yasin, D. I. Gorbunov, S. Zherlitsyn, H. Nakotte, K. Kothapalli, F. Nasreen, C. Cupp, H. B. Cao, A. Kolomiets, and L. Havela, $\mathrm{U}_{2} \mathrm{Ni}_{2} \mathrm{Sn}$ and the origin of magnetic anisotropy in uranium compounds, Phys. Rev. B 99, 064415 (2019).

[10] L. C. J. Pereira, J. A. Paixão, P. Estrela, M. Godinho, F. Boudarot, M. Bonnet, J. Rebizant, J. C. Spirlet, and M. Almeida, A single-crystal magnetization and neutron scattering investigation of the magnetic structure of $\mathrm{U}_{2} \mathrm{Rh}_{2} \mathrm{Sn}$, J. Phys.: Condens. Matter 8, 11167 (1996).

[11] H. Nakotte, A. Purwanto, R. A. Robinson, K. Prokeš, J. C. P. Klaasse, P. F. de Châtel, F. R. de Boer, L. Havela, V. Sechovský, L. C. J. Pereira, A. Seret, J. Rebizant, J. C. Spirlet, and F. Trouw, Hybridization effects in $\mathrm{U}_{2} T_{2} X$ compounds: Magnetic structures of $\mathrm{U}_{2} \mathrm{Rh}_{2} \mathrm{Sn}$ and $\mathrm{U}_{2} \mathrm{Ni}_{2} \mathrm{In}$, Phys. Rev. B 53, 3263 (1996).

[12] K. Prokeš, D. I. Gorbunov, M. Reehuis, B. Klemke, A. Gukasov, K. Uhlî́rová, X. Fabrèges, Y. Skourski, F. Yokaichiya, S. Hartwig, and A. V. Andreev, Anisotropic physical properties of single-crystal $\mathrm{U}_{2} \mathrm{Rh}_{2} \mathrm{Sn}$ in high magnetic fields, Phys. Rev. B 95, 174433 (2017).

[13] M. N. Peron, Y. Kergadallan, J. Rebizant, D. Meyer, S. Zwirner, L. Havela, H. Nakotte, J. C. Spirlet, G. M. Kalvius, E. Colineau,
J. L. Oddou, C. Jeandey, J. P. Sanchez, and J. M. Winand, A new family of actinide ternary intermetallic compounds, J. Alloys Compd. 201, 203 (1993).

[14] L. Havela, V. Sechovský, P. Svoboda, M. Diviš, H. Nakotte, K. Prokeš, F. R. de Boer, A. Purwanto, R. A. Robinson, A. Seret, J. M. Winand, J. Rebizant, J. C. Spirlet, M. Richter, and $\mathrm{H}$. Eschrig, Heavy fermion behavior of $\mathrm{U}_{2} \mathrm{~T}_{2} X$ compounds, $\mathrm{J}$. Appl. Phys. 76, 6214 (1994).

[15] A. M. Strydom, P. de V. du Plessis, and V. V. Gridin, Lowtemperature transport properties of $\mathrm{U}_{2} \mathrm{Rh}_{2} \mathrm{Sn}$ and $\mathrm{U}_{2} \mathrm{Fe}_{2} \mathrm{Sn}$, Phys. B 225, 89 (1996).

[16] V. H. Tran, Z. Zolnierek, A. J. Zaleski, and H. Noël, A. C.magnetic susceptibility study of $\mathrm{U}_{2} T_{2} \mathrm{M}(T=\mathrm{Ni}, \mathrm{Rh}, \mathrm{Pd}$, Ir and Pt, $M=$ In or Sn), Solid State Commun. 101, 709 (1997).

[17] H. Nakotte, K. Prokeš, E. Brück, N. Tang, F. R. de Boer, P. Svoboda, V. Sechovský, L. Havela, J. M. Winand, A. Seret, J. Rebizant, and J. C. Spirlet, High-field magnetization of $\mathrm{U}_{2} \mathrm{~T}_{2} X$ compounds ( $T=\mathrm{Co}, \mathrm{Ni}, \mathrm{Rh}, \mathrm{Pd}, \mathrm{Ir}, \mathrm{Pt}$ and $X=\mathrm{In}, \mathrm{Sn})$, Physica B 201, 247 (1994).

[18] T. Fukushima, S. Matsuyama, T. Kumada, K. Kindo, K. Prokeš, H. Nakotte, F. R. de Boer, L. Havela, V. Sechovský, J. M. Winand, J. Rebizant, and J. C. Spirlet, High-field magnetization studies of some $\mathrm{U}_{2} \mathrm{~T}_{2} X$ compounds, Physica B 211, 142 (1995).

[19] F. R. de Boer, K. Kindo, H. Nakotte, K. Prokeš $\AA_{i}$, and V. Sechovský, $\mathrm{U}_{2} \mathrm{~T}_{2} X(T=\mathrm{Co}, \mathrm{Ni}, \mathrm{Rh}, \mathrm{Pd}, \mathrm{Ir}, \mathrm{Pt} ; X=\mathrm{In}$, Sn) compounds in high magnetic fields, Physica B 246, 129 (1998).

[20] B. Lüthi, Physical Acoustics in the Solid State (Springer, Heidelberg, 2005).

[21] S. Zherlitsyn, S. Yasin, J. Wosnitza, A. A. Zvyagin, A. V. Andreev, and V. Tsurkan, Spin-lattice effects in selected antiferromagnetic materials (Review Article), Low Temp. Phys. 40, 123 (2014).

[22] Y. Skourski, M. D. Kuz'min, K. P. Skokov, A. V. Andreev, and J. Wosnitza, High-field magnetization of $\mathrm{Ho}_{2} \mathrm{Fe}_{17}$, Phys. Rev. B 83, 214420 (2011).

[23] T. Akazawa, T. Suzuki, H. Goshima, T. Tahara, T. Fujita, T. Takabatake, and H. Fujii, Quadrupolar and magnetic phase diagram of UNiSn, J. Phys. Soc. Jpn. 67, 3256 (1998).

[24] T. Suzuki, I. Ishii, N. Okuda, K. Katoh, T. Takabatake, T. Fujita, and A. Tamaki, Quadrupolar ordering of $5 f$ electrons in $\mathrm{UCu}_{2} \mathrm{Sn}$, Phys. Rev. B 62, 49 (2000).

[25] S. I. Fujimori, Y. Saitoh, T. Okane, A. Fujimori, H. Yamagami, Y. Haga, E. Yamamoto, and Y. Ōnuki, Itinerant to localized transition of $f$ electrons in the antiferromagnetic superconductor $\mathrm{UPd}_{2} \mathrm{Al}_{3}$, Nat. Phys. 3, 618 (2007).

[26] B. Lüthi, in Dynamical Properties of Solids, edited by G. K. Horton and A. A. Maradudin (North-Holland, Amsterdam, 1980), Vol. 3.

[27] N. V. Hieu, T. Takeuchi, H. Shishido, C. Tonohiro, T. Yamada, H. Nakashima, K. Sugiyama, R. Settai, T. D. Matsuda, Y. Haga, 
M. Hagiwara, K. Kindo, S. Araki, Y. Nozue, and Y. Onuki, Magnetic properties and crystalline electric field scheme in $R \operatorname{RhIn}_{5}$ (R: rare earth), J. Phys. Soc. Jpn. 76, 064702 (2007).

[28] M. T. Hutchings, Point-charge calculations of energy levels of magnetic ions in crystalline electric fields, Solid State Phys. 16, 227 (1964).

[29] D. I. Gorbunov, T. Nomura, I. Ishii, M. S. Henriques, A. V. Andreev, M. Doerr, T. Stöter, T. Suzuki, S. Zherlitsyn, and J.
Wosnitza, Crystal-field effects in the kagome antiferromagnet $\mathrm{Ho}_{3} \mathrm{Ru}_{4} \mathrm{Al}_{12}$, Phys. Rev. B 97, 184412 (2018).

[30] D. I. Gorbunov, I. Ishii, T. Nomura, M. S. Henriques, A. V. Andreev, M. Uhlarz, T. Suzuki, S. Zherlitsyn, and J. Wosnitza, Magnetic phase diagram and crystal-field effects in the kagomelattice antiferromagnet $\mathrm{U}_{3} \mathrm{Ru}_{4} \mathrm{Al}_{12}$, Phys. Rev. B 99, 054413 (2019).

[31] http://www.mgml.eu. 